\title{
Características técnico-económicas de los sistemas de producción bovina basados en razas criollas introducidas en México
}

\section{Technical-economic characteristics of bovine production systems based on creole breeds introduced in Mexico}

\author{
Rosa Inés Parra-Cortés 1 [0, Miguel Ángel Magaña-Magaña ${ }^{1 *}$ (이 \\ ${ }^{1}$ Tecnológico Nacional de México/I.T-Conkal. División de Estudios de Posgrado e Investigación. Avenida Tecnológico s/n, CP. 97345. \\ Conkal, Yucatán, México. \\ *Autor de correspondencia: drmmagana@gmail.com
}

Artículo científico recibido: 26 de febrero de 2019 aceptado: 31 de julio de 2019

RESUMEN. El objetivo del estudio fue generar información técnico-económica relevante de los sistemas de producción bovina basados en razas criollas introducidas en zonas tropicales de México. Para lo cual se realizó una encuesta por muestreo aleatorio a productores que crían ejemplares Romosinuano y Lechero Tropical en los estados de San Luis Potosí, Veracruz, Tabasco, Campeche, Yucatán y Chiapas. Los productores tuvieron una edad promedio de 53 años, nivel de escolaridad superior y experiencia en la actividad ganadera por 24 años. Los sistemas de producción fueron de vocación pastoril y los parámetros de desempeño de la raza Romosinuano fueron peso al nacimiento y destete de 29 y $172 \mathrm{~kg}, 83 \%$ de fertilidad y 426 días de intervalo entre partos. Estos parámetros fueron para la raza Lechero Tropical de 28 y $178 \mathrm{~kg}$, $81 \%$ y 389 días, respectivamente. Asimismo, se destaca para hembras de vientre una distribución por genotipo de $46.4 \%$ puro, $26.9 \%$ media sangre y $26.7 \%$ otras cruzas, para sementales fue de $98 \%$ puro y $2 \%$ media sangre. Se constató que los productores tienen un control limitado de la información técnico-administrativa, condición que puede ser superada a través de la adopción de buenas prácticas ganaderas y de gestión empresarial. En términos generales, las razas criollas presentan ventajas zootécnicas que se traducen en mayor rentabilidad de los sistemas de producción y las convierten en alternativa viable para cruzamientos con otras razas, ya que pueden transferir genes de adaptabilidad al medio tropical, por lo que su conservación debe ser priorizada.

Palabras clave: Ganadería, gestión empresarial, recurso zoogenético, sostenibilidad, trópico

ABSTRACT. The objective of this study was to generate relevant technical-economic information of the bovine production systems based on creole breeds introduced in tropical zones of Mexico. For which a random sampling survey was carried out to producers who breed Romosinuano and Lechero Tropical specimens in the states of San Luis Potosi, Veracruz, Tabasco, Campeche, Yucatan and Chiapas. The producers had an average age of 53 years, a higher level of education and experience in livestock activity for 24 years. The production systems were of pastoral vocation and the parameters of performance of the Romosinuano breed were weight at birth and weaning of 29 and $172 \mathrm{~kg}, 83 \%$ fertility and 426 days of interval between births; these parameters were for the Lechero Tropical breed of 28 and $178 \mathrm{~kg}, 81 \%$ and 389 days, respectively. Likewise, stands out for adult cows a distribution by genotype of $46.4 \%$ pure, $26.9 \%$ medium blood and $26.7 \%$ other crosses, for adult bulls was $98 \%$ pure and $2 \%$ half blood. It was found that producers have limited control of technical-administrative information, a condition that can be overcome through the adoption of good livestock practices and business management. In general terms, that creole breeds have zootechnical advantages that result in higher profitability of production systems and make them a viable alternative for crosses with other breeds, since they can transfer genes of adaptability to the tropical environment, which is why its conservation must be prioritized.

Key words: Livestock, business management, animal genetic resources, sustainability, tropics 


\section{INTRODUCCIÓN}

La creciente demanda de alimentos de origen animal ha masificado la cría de ganados en países en vías de desarrollo, suceso conocido como revolución ganadera y caracterizado por la intensificación de los sistemas de producción animal (Thornton 2010, Wright et al. 2012). En América Latina, el desarrollo de actividades ganaderas intensivas, provocó la introducción de razas bovinas de alto potencial productivo, pero provenientes en su mayoría de países localizados en zonas templadas. Posteriormente, se presentaron cruzamientos genéticos indiscriminados, asociados a la desaparición gradual de las razas locales. A pesar de la contribución que hace la intensificación de la ganadería a la oferta de alimentos, crece la preocupación por la pérdida de recursos zoogenéticos, considerados necesarios para satisfacer las necesidades humanas (FAO 2015, Zavala 2019).

En este grupo de recursos se incluye a los bovinos criollos, que descienden de los primeros bovinos Bos taurus que llegaron al continente americano en 1493, procedentes de la Península Ibérica (Primo 1992). Para el caso de México se reporta que estos bovinos arribaron al puerto de Veracruz en 1521 y en las costas de Campeche en 1543 (De Alba 2011). Posteriormente, estos bovinos se cruzaron y evolucionaron bajo diversas condiciones climáticas y de alimentación; procesos que, aunados al aislamiento geográfico y la selección de animales de forma natural, contribuyeron a la formación de grupos raciales. En la actualidad, en México las razas criollas de bovinos que se preservan son de Baja California Sur, localizada en las Costas de Sonora, Sinaloa y en la Península de Baja California; de Chihuahua o de Rodeo en el norte del país; del Golfo en los llanos de la Costa de Veracruz; de la Sierra Madre Occidental en los estados de Durango, Jalisco, Nayarit y Zacatecas; de la Mixteca o Poblana en Oaxaca, Guerrero y Puebla; de Chiapas en las sierras del estado del mismo nombre y de Nunkiní en la Península de Yucatán (Méndez et al. 2002, Hernández et al. 2015, Villaseñor et al. 2017). También se han introducido en las zonas cálidas ejemplares de las razas Romosinuano y Lechero tropical, procedentes de hatos localizados en Estados Unidos y Costa Rica, pero con origen genético en Colombia y Nicaragua, respectivamente (De Alba 2011). El estado actual del inventario bovino de razas criollas se desconoce, pero informes señalan que los ejemplares de este tipo de ganado se encuentran en peligro de extinción, debido a la reducción de sus núcleos genéticos por cruzamientos indiscriminados con otras razas lecheras de origen europeo, y en otros casos cárnicas de tipo cebuino (FAO 2015).

Con respecto a la ganadería de México, la disponibilidad de recursos naturales derivada de la gran diversidad climática del país, ha favorecido el desarrollo de distintos sistemas de producción, desde tradicionales con limitado nivel de tecnología y de bajo costo, hasta los especializados con tecnologías de punta y alto costo (Rojo-Rubio et al. 2008). Los sistemas con mayor presencia son los tradicionales, mismos que varían en tamaño del hato, finalidad productiva, manejo tecnológico y condición socioeconómica (Hernández et al. 2013, Rodríguez et al. 2018). También se reporta para zonas tropicales de México que predominan los sistemas de doble propósito, principalmente extensivos y de bajo costo. Pero por las exigencias del mercado internacional crece el interés de los productores por razas cárnicas orientadas a la obtención de becerros para la venta (Magaña et al. 2006). En el 2017, el inventario nacional bovino ascendió a 31.9 millones de cabezas, conformado principalmente por vacas para cría de becerros $(29.9 \%)$, animales en desarrollo $(24.5 \%)$, vaquillas para remplazo (14.5\%), animales en engorda (14\%) y vacas para cría de becerros y ordeña (8.8\%), entre otras categorías. Con relación a la participación por estado, destaca el aporte al inventario bovino: Veracruz con $14 \%$, Jalisco $11 \%$, Tabasco $8 \%$, así como Chihuahua y Chiapas con $7 \%$ cada uno (INEGI 2017). A partir de este inventario bovino se reportó una producción nacional de 11.8 millones de toneladas de leche y 1.9 millones de toneladas de carne (FAO 2019).

En el ámbito general, la ganadería se enfrenta a riesgos del entorno, que influyen sobre el desempeño de los sistemas de producción, condición que se agudiza con los efectos asociados al cambio climático 
(Parra et al. 2019). Por lo que es imperante los cuestionamientos sobre la baja eficiencia y rentabilidad de los sistemas de producción bovina en el trópico (López-Vigoa et al. 2017). Por lo que en la búsqueda de alternativas de mejora es necesario realizar el análisis sistémico de la actividad ganadera, como el cruce de razas criollas con otras razas de mayor inventario, lo que puede contribuir a la sostenibilidad técnica, económica, social y ambiental de los sistemas de producción (Parra-Cortés et al. 2019). Pero, solo el $16.3 \%$ del inventario bovino de México está constituido por animales cruzados, corrientes y criollos, pero se desconoce el total de cabezas que corresponde a estos últimos (INEGI 2017). Además, es escasa la información sobre el desempeño y eficiencia de los bovinos criollos que han sido introducidos en México. Por lo que, el objetivo fue generar información sobre los factores técnicos y económicos de sistemas de producción bovina para ejemplares de razas criollas introducidas en las regiones tropicales de México; para contribuir al conocimiento y posicionamiento de estos recursos zoogenéticos para la mejora de la producción ganadera.

\section{MATERIALES Y MÉTODOS}

\section{Localización y descripción del área de estudio}

El estudio se realizó en la región tropical que comprende las llanuras y lomeríos de las costas del Golfo de México, las llanuras de la Península de Yucatán y los valles de las Sierras de Chiapas (Figura 1). La región seleccionada incluye zonas con clima cálido húmedo con rango de precipitación anual de 2000 a $4000 \mathrm{~mm}$, así como zonas de tipo subhúmedo que reportan entre 1000 a $2000 \mathrm{~mm}$ de precipitación anual; con temperatura entre 22 y $26{ }^{\circ} \mathrm{C}$ (INEGI 2019).

\section{Universo de estudio}

Se consideró a los productores vinculados a la Asociación Mexicana de Criadores de Ganado Romosinuano y Lechero Tropical (AMCROLET). Al comité directivo de esta organización se le solicitó los criterios de clasificación de los sistemas de producción bovina según la finalidad productiva. Pos- teriormente se realizó un listado de productores de esta asociación con hatos localizados en el área de estudio, seleccionando aquellos que gestionan sistemas de producción con visión empresarial; integrándose una lista de 49 productores.

\section{Obtención de la información}

La información base del estudio se obtuvo entre mayo y diciembre de 2018, para lo cual se realizó una encuesta por muestreo estadístico a productores de bovinos criollos ubicados en el área de estudio. Se realizaron visitas de campo a las unidades de producción para la aplicación de la cédula de entrevista a productores o encargados de las mismas; también se corroboró la información proporcionada por los entrevistados por observación directa.

\section{Entrevista}

Con este instrumento se obtuvo información sobre manejo zootécnico y aspectos socioeconómicos relacionados con la cría de ganado bovino. La entrevista se estructuró con 134 preguntas, con secciones de datos generales, inventarios, alimentación, reproducción, genética, sanidad, manejo de praderas, rutinas de manejo, asistencia técnica, recursos naturales, comercialización e información adicional del productor. La entrevista fue previamente evaluada por investigadores del área de conocimiento y se probó en campo con 10 productores, para corregir deficiencias y determinar su funcionalidad al llenado.

\section{Muestreo}

Con el fin de obtener la validez estadística de la información obtenida se optó por un muestreo irrestricto aleatorio para estimación de proporciones, de acuerdo al procedimiento propuesto por Scheaffer et al. (1987). Para lo cual se consideró la probabilidad de selección de los sistemas de producción bovina por finalidad productiva, las cuales fueron doble propósito y vocación cárnica; el marco de muestreo se integró con la lista de productores proporcionada por AMCROLET y se contempló como unidad de producción de interés aquellas que poseían hatos de bovinos criollos de 30 animales o más. La muestra 


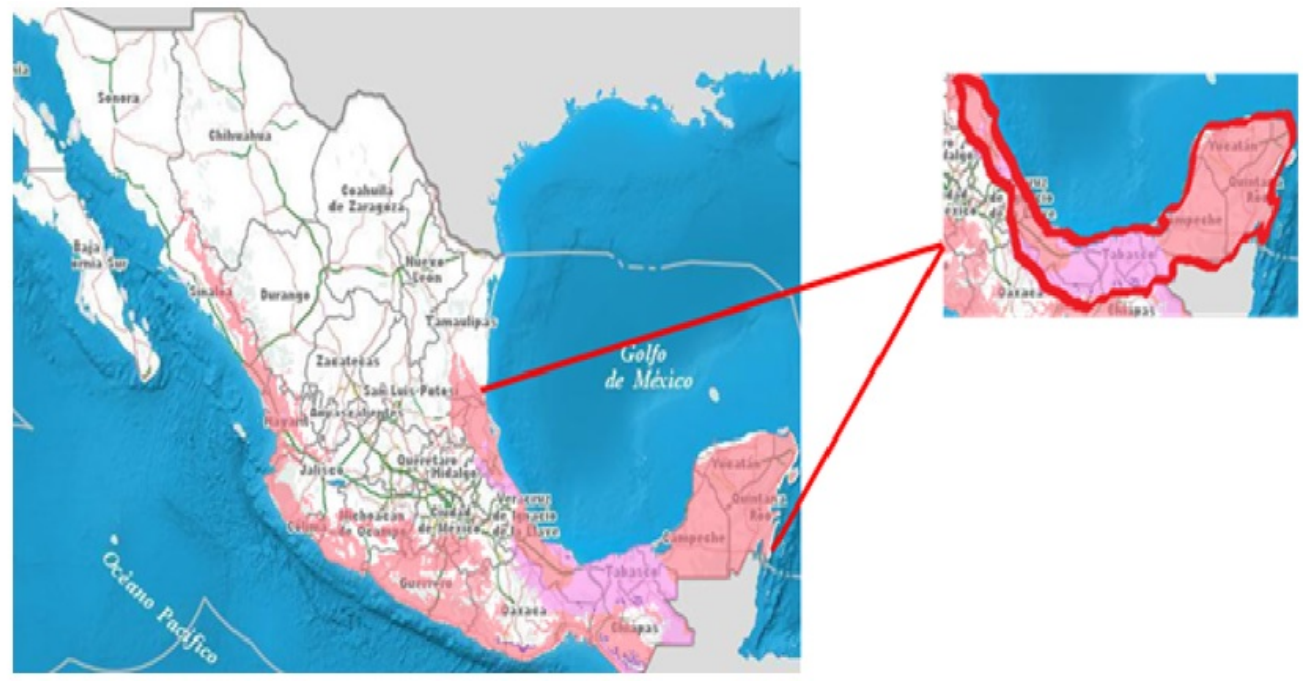

Figura 1. Zonas tropicales de México y localización del área de estudio.

preliminar fue elegida completamente al azar y se constituyó por 33 productores. Como error de estimación (B) se asumió el 10\% del valor promedio de la probabilidad de que un sistema perteneciera a una de las dos finalidades productivas. Para determinar el número final de productores a entrevistar (n) se utilizó la siguiente formula:

$$
n=\frac{N p q}{(N-1) D+p q}
$$

Dónde: $\mathrm{N}=$ Número total productores en los listados, $p=$ Probabilidad finalidad el doble propósito, $q=$ Probabilidad finalidad la vocación cárnica y $D=B^{2} / 4$; donde $\mathrm{B}$ es el error de estimación.

\section{Sistematización y análisis de los datos}

La información obtenida en campo se registró en una base de datos, en una hoja de cálculo del programa informático Excel versión 2019, organizando las secciones de información en variables de interés. La base de datos con la información de las entrevistas se revisó con el fin de detectar incongruencias en la información obtenida, validando la base de datos para garantizar la objetividad del análisis. Los parámetros asociados al desempeño productivo de los hatos fueron estimados mediante estadística descripta, a partir de las variables consignadas en la base de datos que se conformó y empleando las rutinas de cálculo propias para los descriptores correspondientes.

La estimación de los costos de producción e ingresos se consideraron los valores anuales de adquisición de insumos, mano de obra, depreciación de activos, otros gastos y ventas. La rentabilidad del sistema de producción se calculó al restar el costo total de producción al valor de las ventas, el respectivo índice se obtuvo al dividir el valor de la rentabilidad entre el costo de producción contabilizados por sistema de producción de interés. Los valores monetarios de los parámetros estimados se presentan en dólares americanos (USD) empleando una tasa de cambio representativa del mercado que correspondió $\$ 19.10$ del Banco de México; los parámetros de comparación lo constituyen valores promedio anualizados por vientre y unidad de superficie de cada sistema de producción identificado en el área de estudio.

\section{RESULTADOS}

La superficie promedio de las unidades de producción estudiadas fue de 188.6 hectáreas, con rango de 20 a 508 ha; de las que se destina el $67.4 \%$ a praderas en su mayoría de Brachiaria, Cynodon y Panincum, $1.2 \%$ a cultivos forrajeros de corte (Penni- 
setum purpureum), $4.7 \%$ a silvopastoreo (Leucaena y Guazuma), 24.7\% a reserva forestal y, el área restante con construcciones, cultivos agrícolas diversos y reservorios de agua. La tenencia de la tierra de las unidades es de propiedad privada en $87 \%$, arrendamiento el $9 \%$ y propiedad ejidal el $4 \%$.

En el área de estudio no se encontró sistemas de producción bovina propiedad o gestionados por mujeres. Las principales variables socioeconómicas de los productores son edad promedio de 53 años, escolaridad de 16 años y experiencia en la actividad ganadera de 24 años. El 65\% de los productores consideran a la ganadería como actividad económica secundaria, condición que puede estar asociada con su nivel de escolaridad que les permite integrarse al mercado laboral como mano de obra calificada, por lo que la cría de bovinos es una oportunidad de ahorro $y$ de ingresos adicionales.

El inventario animal de las unidades de producción tiene un rango de 10 a 572 cabezas, con una media de 208.8 y desviación estándar de 163.8 cabezas. El inventario total fue de 4803 cabezas, con estructura del hato ganadero conformada por $1.7 \%$ de sementales, $30.6 \%$ de vacas, $27.9 \%$ de novillonas, $4.9 \%$ de machos en desarrollo y toretes, $19.6 \%$ de becerras y $15.3 \%$ de becerros. Por otra parte, y con relación con las razas criollas, el $65 \%$ de los sistemas de producción mantienen ejemplares de la raza Romosinuano y el 35\% la raza Lechero Tropical.

Se identificaron cuatro categorías de unidades de producción por finalidad productiva, mismas que son doble propósito, venta de destetes, venta de pie de cría y multipropósito (Tabla 1). En la primera finalidad se obtiene leche como principal producto para el mercado y, en forma complementaria, se venden los terneros destetados; en la segunda, se ofertan terneros destetados para engorda en otros ranchos, especialmente los localizados en el norte del país. La tercera produce hembras y machos con alto valor genético, que son comercializados para establecer nuevos núcleos y planes de mejoramiento genético; la última finalidad, considerada fue multipropósito dedicada a la producción de leche, venta de destetes y animales para pie de cría.
Tabla 1. Distribución de sistemas de producción según finalidad productiva y raza.

\begin{tabular}{|c|c|c|c|}
\hline \multirow{2}{*}{$\begin{array}{l}\text { Finalidad } \\
\text { productiva }\end{array}$} & \multirow{2}{*}{$\begin{array}{c}\text { Frecuencia } \\
\text { (\%) }\end{array}$} & \multicolumn{2}{|c|}{$\begin{array}{c}\text { Proporción de animales } \\
\text { por raza (\%) }\end{array}$} \\
\hline & & Romosinuano & $\begin{array}{l}\text { Lechero } \\
\text { tropical }\end{array}$ \\
\hline Doble propósito & 13 & - & 100 \\
\hline Venta de destetes & 57 & 85 & 15 \\
\hline Venta de pie de cría & 17 & 75 & 25 \\
\hline Multipropósito & 13 & 33 & 67 \\
\hline
\end{tabular}

Las principales prácticas de manejo de los productores, fueron el control preventivo de enfermedades con la aplicación de vacunas, para la rabia paralítica bovina o derriengue (65\%), Leptospirosis $(13 \%)$ y bacterianas ocasionadas por los géneros Clostridium y Pasteurella; práctica común de todos los productores entrevistados. Para el control de ectoparásitos como moscas y garrapatas, el $65 \%$ de los productores realizan baños de aspersión con productos biológicos con frecuencia de aplicación variable, según las condiciones climáticas y el nivel de infestación. Para el control de endoparásitos como anaplasmas, babesias y coccidias, el $38 \%$ de los productores prefieren usar fármacos orales con intervalos de aplicación trimestrales, cuatrimestrales o semestrales, según el grado de afectación de los animales. El $17 \%$ de las unidades de producción tuvieron animales con lesiones en la piel, padecimiento relacionado con exposición excesiva al sol, característica de pelo corto propia de razas criollas de zonas tropicales, así como perdida de pelo por edad avanzada. Las lesiones en la piel en los bovinos criollos reportadas por los productores se agudizan en las épocas de canícula y seca, lo que coincide con la mayor infestación de ectoparásitos como garrapatas y moscas. La mortalidad en los hatos no superó el $3 \%$ del total del inventario animal que los productores mantienen en sus predios durante el año.

Los sistemas de producción de la muestra fueron de tipo pastoril, con suministro de alimento balanceado a becerros y vacas en período de lactancia correspondió al 13 y $17 \%$ de los casos, respectivamente. Mientras que el $78 \%$ de los entrevistados suministran a sus animales sal mineralizada de tipo comercial de forma continua y el restante lo restringe a épocas secas. En cuanto, al manejo genético se ob- 
servó que los criterios de selección y cruzamiento de los ejemplares fueron determinados por los productores, a partir de la observación directa de la apariencia externa y actitud productiva de los individuos que conforman el hato. Los productores tienen carencia de planes de mejoramiento genético oficiales para las razas Romosinuano y Lechero tropical. Los sistemas de pastoreo son rotacionales (52\%), silvopastoriles $(26 \%)$ e intensivos (22\%), presentando los últimos un mayor número de áreas de pastoreo con cercas móviles. El $83 \%$ de los productores orientan la asistencia técnica al chequeo reproductivo de las hembras de vientre que conforman sus hatos, así como en menor proporción al manejo de la sanidad, nutrición y genética del hato con médicos veterinarios de la región (Figura 2).

En la Tabla 2 se indican los principales parámetros de desempeño animal reportados por los productores. El genotipo de las hembras de vientre en los hatos presentó una distribución de $46.4 \%$ puro, $26.9 \%$ media sangre y $26.7 \%$ otras cruzas, y para los sementales fue de $98 \%$ puro y $2 \%$ media sangre. También se observó que el $50 \%$ de los sistemas de producción basados en la raza criolla Lechero Tropical realizan ordeño de tipo mecánico con producción promedio diaria de $6.55 \pm 1.68 \mathrm{~L}$ de leche por vaca.

Por la superficie del predio, tamaño del hato y el nivel de escolaridad de los productores, los sistemas de producción de interés se pueden categorizar como de visión empresarial. Para el componente administrativo se observó que todos los sistemas no implementan organigramas y registros contables, por lo que se lleva a cabo una limitada gestión estratégica. En cuanto a la procedencia del personal operativo, se tiene de tipo permanente y temporal, que se encuentra contratado de forma informal, con pago por jornales laborados que se pagan de forma semanal.

El manejo de registros de información en los sistemas de producción, se realiza de forma limitada, así en la muestra se observó anotaciones de campo sobre nacimientos de crías, pesajes y ventas; mientras que el $43 \%$ de los casos utilizó un software especializado para el control del inventario de animales. Los productores o los encargados de la unidad de producción realizan anotaciones en libretas de campo, en especial sobre el nacimiento de crías y algunos parámetros productivos como peso al destete. No se encontró evidencia de seguimiento administrativo eficiente, ya que en el aspecto operativo se registran datos como la compra de insumos; solo el $30.3 \%$ de los productores llevan algunos registros de información a través de hojas de cálculo electrónico. Lo que evidencia una limitada gestión empresarial en los sistemas de producción bovina basados de razas criollas. En tanto, que la comercialización de animales para la engorda y leche, así como la adquisición de los insumos se realizan en mayor proporción en el ámbito regional.

Los productos comercializados tienen los siguientes rangos de precio: de USS $\$ 0.29$ a 0.31 el litro de leche, de US\$2.23 a 2.77 el kilogramo de ternero destetado; de US\$1571 a 2094 por animal para pie de cría y, de US\$1.15 a 1.41 USD el kilogramo de animal de desecho. Los precios de los dos primeros y del último producto, son determinados por el comportamiento del mercado regional y nacional, mientras que en el caso del tercer producto el precio lo determina el productor y, en menor proporción, se fija por acuerdo mutuo entre el comprador y vendedor.

La estructura de costos de producción se constituyó por costos variables con un rango de 58 a $69 \%$ y la proporción restante correspondió a costos fijos. Se destaca una mayor participación del rubro alimentación en los sistemas de doble propósito, el cual representó el 39\% del costo total de producción, que correspondió a la adquisición y suministro de alimento balanceado principalmente a las hembras en lactancia. Asimismo, la mano de obra registró un rango de 22 a $35 \%$ del costo total, mientras que los rubros de medicamentos e insumos veterinarios, combustibles, servicios públicos y otros gastos presentaron una baja participación porcentual (Tabla 3).

\section{DISCUSIÓN}

En México, la ganadería criolla posee un papel socioeconómico y ecológico importantes en diversas regiones y se asocia a poblaciones rurales de bajos recursos económicos (Alonso y Ulloa 1997). Pero 


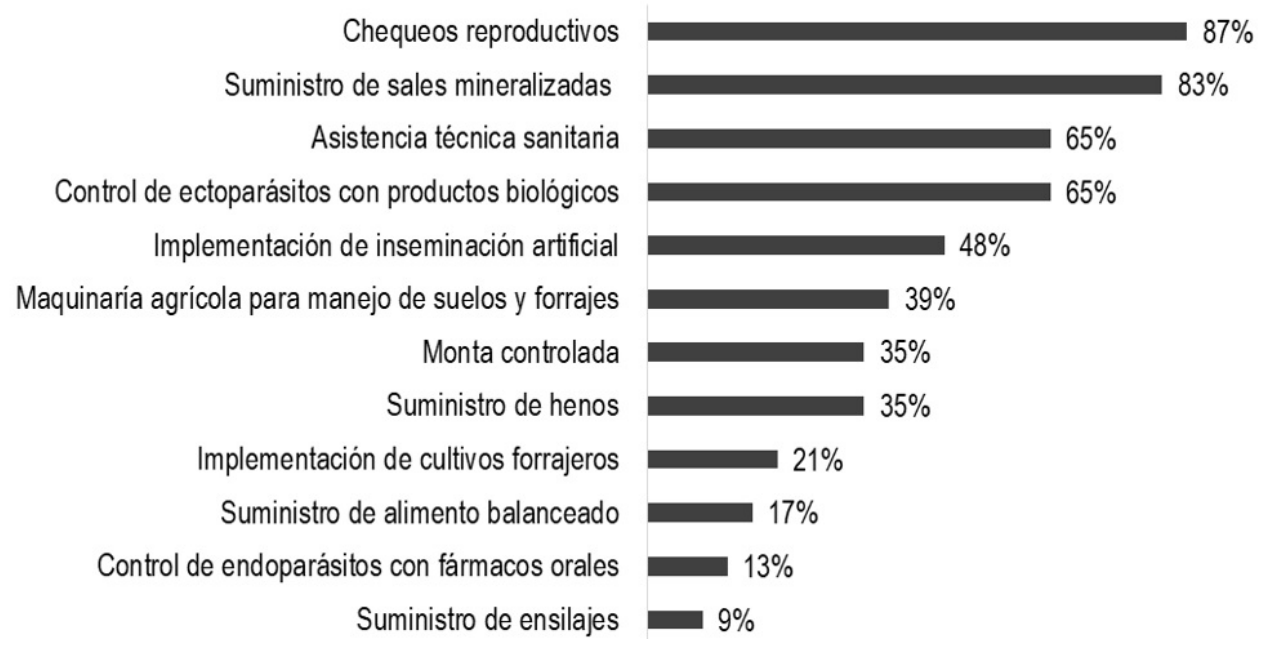

Figura 2. Prácticas de manejo en los sistemas de producción bovina.

Tabla 2. Principales parámetros de desempeño animal por finalidad productiva y tipo racial.

\begin{tabular}{|c|c|c|c|c|c|c|}
\hline \multirow[b]{2}{*}{ Parámetro } & \multicolumn{4}{|c|}{ Finalidad productiva } & \multicolumn{2}{|c|}{ Tipo racial } \\
\hline & $\begin{array}{c}\text { Doble } \\
\text { propósito }\end{array}$ & $\begin{array}{l}\text { Venta de } \\
\text { destete }\end{array}$ & $\begin{array}{c}\text { Venta de } \\
\text { pie de cría }\end{array}$ & Multipropósito & Romosinuano & $\begin{array}{l}\text { Lechero } \\
\text { Tropical }\end{array}$ \\
\hline Peso al nacimiento $(\mathrm{kg})$ & $28 \pm 3$ & $29 \pm 1$ & $29 \pm 1$ & $29 \pm 1$ & $29 \pm 1$ & $28 \pm 2$ \\
\hline Peso al destete $(\mathrm{kg})$ & $179 \pm 2$ & $175 \pm 15$ & $177 \pm 6$ & $167 \pm 15$ & $172 \pm 15$ & $178 \pm 4$ \\
\hline Edad al destete (días) & $230 \pm 17$ & $222 \pm 23$ & $230 \pm 17$ & $220 \pm 17$ & $219 \pm 22$ & $233 \pm 14$ \\
\hline Tasa de fertilidad (\%) & $78 \pm 8$ & $83 \pm 5$ & $83 \pm 12$ & $83 \pm 6$ & $83 \pm 5$ & $81 \pm 8$ \\
\hline Tasa de natalidad (\%) & $85 \pm 5$ & $86 \pm 13$ & $84 \pm 5$ & $85 \pm 5$ & $85 \pm 12$ & $85 \pm 5$ \\
\hline Intervalo entre partos (días) & $387 \pm 38$ & $432 \pm 94$ & $393 \pm 49$ & $377 \pm 20$ & $426 \pm 94$ & $389 \pm 28$ \\
\hline Días abiertos & $104 \pm 38$ & $147 \pm 96$ & $110 \pm 49$ & $94 \pm 20$ & $146 \pm 94$ & $100 \pm 26$ \\
\hline Edad al 1.er servicio (meses) & $19 \pm 5$ & $24 \pm 1$ & $21 \pm 5$ & $23 \pm 2$ & $23 \pm 2$ & $22 \pm 4$ \\
\hline Peso 1.er servicio (kg) & $213 \pm 23$ & $238 \pm 13$ & $227 \pm 23$ & $233 \pm 12$ & $235 \pm 16$ & $228 \pm 18$ \\
\hline Edad 1.er parto (meses) & $28 \pm 5$ & $33 \pm 1$ & $30 \pm 5$ & $32 \pm 2$ & $32 \pm 2$ & $31 \pm 4$ \\
\hline
\end{tabular}

hay evidencias que permiten asociar las razas criollas bovinas a sistemas de producción con vocación empresarial. En este sentido, entre las características físicas de las unidades de producción, destaca el tamaño promedio del predio, el cual fue similar al reportado para el nivel nacional por el programa de estímulos a la productividad ganadera para predios de productores tipo IV, es decir con una superficie de 110 hectáreas, caracterizados por ejercer un mayor manejo empresarial (Leos-Rodríguez et al. 2008). Por su parte, para sistemas de doble propósito en las regiones sureste, centro, noroeste y suroeste de México se reportó un tamaño de predio promedio de 55, 49.3, 88 y 59 hectáreas, respectivamente (OsorioArce et al. 1999, Hernández et al. 2013, AlbarránPortillo et al. 2015, Cuevas-Reyes y Rosales-Nieto
2018). El tamaño de predio en estas regiones fue inferior a la muestra, pero para el estado de Chiapas se determinó un valor superior de 142 hectáreas (Bautista-Martínez et al. 2019). Para el caso de los sistemas de producción bovina localizados en Sinaloa, se determinó una relación directa entre el tamaño del predio y el potencial comercial de la empresa ganadera (Cuevas-Reyes et al. 2017). De igual manera, se observó que el tamaño de los predios destinados a la cría de bovinos criollos de las razas Romosinuano y Lechero Tropical, está supeditado a la visión empresarial de los productores.

Entre las características socioeconómicas de los productores, destacan la edad y el nivel de escolaridad, así como la experiencia en la actividad ganadera con valores similares a los reportados por 
Tabla 3. Indicadores de desempeño económico de los sistemas de producción (valores en USD).

\begin{tabular}{|c|c|c|c|c|c|c|c|c|}
\hline \multirow{2}{*}{ Indicador } & \multicolumn{2}{|c|}{ Doble Propósito } & \multicolumn{2}{|c|}{ Venta de Destete } & \multicolumn{2}{|c|}{ Venta de pie de cría } & \multicolumn{2}{|c|}{ Multipropósito } \\
\hline & $\mu$ & $\Sigma$ & $\mu$ & $\Sigma$ & $\mu$ & $\sigma$ & $\mu$ & $\sigma$ \\
\hline Superficie en ganadería & 98 & 10 & 190 & 138 & 44 & 32 & 91 & 66 \\
\hline Número de vientres & 98 & 26 & 79 & 62 & 133 & 131 & 66 & 54 \\
\hline Ingreso total & $\$ 98630$ & - & $\$ 41564$ & - & $\$ 31000$ & & $\$ 43798$ & - \\
\hline Costo variable & $\$ 55902$ & - & $\$ 21938$ & - & $\$ 14651$ & & $\$ 23227$ & - \\
\hline Mano de obra & $\$ 18179$ & $\$ 20943$ & $\$ 12742$ & $\$ 9025$ & $\$ 7999$ & $\$ 3946$ & $\$ 10785$ & $\$ 5860$ \\
\hline Alimentación & $\$ 31712$ & $\$ 17114$ & $\$ 5117$ & $\$ 3203$ & $\$ 3565$ & $\$ 4675$ & $\$ 8560$ & $\$ 7383$ \\
\hline Medicamentos & $\$ 593$ & $\$ 60$ & $\$ 644$ & $\$ 601$ & $\$ 563$ & $\$ 79$ & $\$ 524$ & \\
\hline Chequeo reproductivo & $\$ 988$ & $\$ 210$ & $\$ 565$ & $\$ 489$ & $\$ 647$ & $\$ 1138$ & $\$ 673$ & $\$ 417$ \\
\hline Combustibles & $\$ 3316$ & $\$ 1683$ & $\$ 2155$ & $\$ 1252$ & $\$ 1113$ & $\$ 752$ & $\$ 1745$ & $\$ 1090$ \\
\hline Mantenimiento & $\$ 908$ & $\$ 242$ & $\$ 644$ & $\$ 360$ & $\$ 707$ & $\$ 262$ & $\$ 873$ & $\$ 121$ \\
\hline Servicios públicos & $\$ 206$ & $\$ 97$ & $\$ 71$ & $\$ 32$ & $\$ 58$ & $\$ 10$ & $\$ 68$ & $\$ 9$ \\
\hline Costo fijo & $\$ 25308$ & - & $\$ 14379$ & - & $\$ 10592$ & - & $\$ 12648$ & - \\
\hline Otros pagos fijos & $\$ 209$ & - & $\$ 209$ & - & $\$ 209$ & - & $\$ 209$ & . \\
\hline Depreciación & $\$ 22140$ & $\$ 22794$ & $\$ 11915$ & $\$ 7272$ & $\$ 6835$ & $\$ 4931$ & $\$ 11124$ & $\$ 8103$ \\
\hline Impuestos & $\$ 2959$ & $\$ 1653$ & $\$ 1247$ & $\$ 770$ & $\$ 930$ & $\$ 521$ & $\$ 1314$ & $\$ 657$ \\
\hline Arrendamiento & - & - & $\$ 1007$ & $\$ 3630$ & $\$ 2618$ & $\$ 5236$ & - & . \\
\hline Costo total & $\$ 81210$ & - & $\$ 36316$ & - & $\$ 25243$ & - & $\$ 35875$ & . \\
\hline Rentabilidad & $\$ 17420$ & - & $\$ 5248$ & - & $\$ 5756$ & - & $\$ 7924$ & . \\
\hline Índice rentabilidad & $28 \%$ & - & $12 \%$ & - & $30 \%$ & - & $24 \%$ & . \\
\hline Rentabilidad ha/año & $\$ 178$ & - & $\$ 28$ & - & $\$ 131$ & - & $\$ 87$ & . \\
\hline Rentabilidad vientre/año & $\$ 177$ & - & $\$ 67$ & - & $\$ 43$ & - & $\$ 121$ & \\
\hline
\end{tabular}

Oros et al. (2011), Orantes-Zebadúa et al. (2014), Albarrán-Portillo et al. (2015) y Cuevas et al. (2016) quienes que reportan un rango de edad de 47 a 56 años y experiencia en ganadería de 24 a 30 años. En los sistemas ganaderos de Estados Unidos, Vance (2013) asoció la edad avanzada de los productores con su capacidad de inversión, condición que explica la adquisición de predios destinados a actividades ganaderas tras varios años de ahorro de capital o el beneficio que reciben los productores al heredar terrenos. Por lo que para el presente estudio se encontró una condición similar, al observarse en términos generales que los productores de bovinos se caracterizan por tener una edad avanzada. La ausencia de relevo generacional en la actividad ganadera es un factor que limita la sostenibilidad; aspectos que no fue abordado en el estudio; por lo cual se sugiere el estudio de esta característica en productores de tipo empresarial de las razas criollas Romosinuano y Lechero Tropical. Por otra parte, se destaca el nivel de escolaridad del productor como variable asociada a la visión empresarial y el grado de especialización del sistema de producción. Al respecto Leos-Rodriguez et al. (2008), reporta que los sistemas de producción bovina tradi- cionales son gestionados por productores con menor escolaridad, mientras los semitecnificados y tecnificados por aquellos con mayor nivel educativo. La muestra se constituyó por sistemas de producción bovina tecnificados, mismos que fueron gestionados por productores con un nivel de escolaridad superior. Los entrevistados manifestaron que su interés por las razas criollas surgió después de acceder a información especializada sobre sus características de adaptación al medio.

Con relación al tamaño y estructura del hato, en Veracruz se reportó un inventario bovino de 6 a 356 cabezas (Vilaboa y Díaz 2009), mientras que en Chiapas las unidades de producción presentaron un promedio de 50 cabezas (Orantes-Zebadúa et al. 2014). Al considerar que las variables tamaño del predio e inventario animal están correlacionadas, el grado de especialización de los sistemas de producción en la muestra, según los criterios propuestos por Rojo-Rubio et al. (2008), estos se pueden considerar como semi-especializados. Con referencia a la estructura del hato en el estado de Yucatán, Osorio-Arce (1999) reportó que ésta se integró por $1.5 \%$ sementales, $60.3 \%$ vacas, $5.5 \%$ novillonas, $8.8 \%$ toretes, $12.5 \%$ becerras y $11.4 \%$ be- 
cerros; mientras que en el estado de Veracruz se observó para estas mismas categorías los porcentajes fueron de $3,50,21,3,12$ y $12 \%$, respectivamente (Vilaboa y Díaz 2009). Se observó un mayor número de hembras jóvenes en la estructura del hato, como resultado de la toma de decisión de los productores por hacer retención de vientres y reemplazo del pie de cría. En este estudio se encontró que el $20 \%$ de los vientres del inventario total, fueron adquiridos por los productores mediante compra en otras unidades de producción, decisión que contribuye a la preservación y multiplicación de los núcleos genéticos de las razas criollas de interés.

Para la variable importancia económica de la cría de bovinos criollos de las razas Romosinuano y Lechero Tropical, se encontró que el 35\% de los productores entrevistados consideraron la cría de bovinos como principal actividad económica, mientras en el estado de Veracruz el $57 \%$ de los productores de razas bovinas convencionales ejerce la ganadería como actividad principal (Vilaboa y Díaz 2009). El menor interés por la cría de bovinos de razas criollas se debe al desconocimiento de los productores sobre las bondades de estas razas, condición atribuida al escaso número de núcleos genéticos que subsisten, principal causa del limitado establecimiento de nuevos hatos de bovinos criollos. Los productores consideraron a la ganadería como su actividad secundaria, ya que, al poseer mayor nivel de escolaridad, tienen la posibilidad de realizar otras actividades remuneradas; por lo que la cría de ganado la consideran como medio de inversión y de ahorro. Otros aspectos relacionados con las características de la familia y la estructura de los ingresos de los productores no fueron suministrados por los entrevistados.

Para los parámetros de desempeño animal de las razas criollas introducidas, se encontró que en sistemas de producción localizados en los estados de Veracruz y Tabasco, la raza Romosinuano tiene un peso promedio al nacimiento de $27.1 \pm 4.9 \mathrm{~kg}$ y al destete es de $147.2 \pm 27.6 \mathrm{~kg}$ a los 240 días (Martínez et al. 2018). Para esta misma raza en Colombia se tiene un peso al nacimiento y al destete de 29.5 y $174 \mathrm{~kg}$ a los 210 días (Martínez 1998), valores que están cercanos a los encontrados en el presente estudio. Por el contrario Ossa et al. (2007a) reporta una edad de 38.21 meses al primer parto en la raza Romosinuano, valor que supera en seis meses a lo que se encontró en el presente estudio. Las diferencias en la edad al primer parto observadas se pueden deber a la interacción genotipo-ambiente y las diversas formas de manejo. Para la vocación zootécnica de la raza Lechero Tropical, se observó que algunos productores han decidido no realizar el ordeño destinado para venta de leche, por efecto de mayor demanda de carne, por lo que prefieren la venta de becerros destetados; cambio de vocación que pone en riesgo la aptitud lechera de la raza. La información sobre los parámetros de crecimiento de los becerros de esta raza son limitados, pero se tienen reportes sobre razas criollas similares productoras de leche en el trópico. Por ejemplo, para la raza Hartón del valle en Colombia se reporta un rango de peso al nacimiento de 31 a $35 \mathrm{~kg}$ y al destete de 165 a $178 \mathrm{~kg}$ a los 245 días de edad (Álvarez 2001), lo que coincide con los valores reportados para los productores entrevistados. Sobre el comportamiento reproductivo de la raza Lechero Tropical se reporta para México, que el primer servicio de las hembras ocurre en promedio a los 20 meses de edad, con peso corporal de 306.8 kilogramos (Severino-Lendechy et al. 2019); valores que son similares a los observados en el presente estudio. Mientras que la edad al primer parto y el intervalo entre partos de esta raza, ocurren en promedio de 40.2 meses y 451 días, respectivamente; valores que son superiores a los reportados por los entrevistados. De manera general, la mayoría de las razas criollas presentan bajo peso al nacimiento, valor que es menor a $30 \mathrm{~kg}$, característica considerada como favorable, ya que es indicador de la capacidad que tienen las vacas de parir terneros sin problemas distócicos (Ossa et al. 2007b). Por lo que, el peso liviano de las crías de hembras criollas implica menores dificultades al momento del parto, lo que genera rápida involución uterina, temprana concepción de nueva preñez y menor intervalo entre partos; lo que repercute en la mayor tasa de fertilidad.

Los ejemplares de la raza Lechero Tropical del país descienden de los criollos centroamericanos, denominados como ganado Reyna. Para esta úl- 
tima raza de ganado, y en condiciones del pacifico nicaragüense, Burgmaier et al. (1999) reportan una producción de leche por vaca de 2.86 litros al día, periodo lactancia de 265 días e intervalo entre partos de 413.7 días, para un sistema de doble propósito. Para la raza Hartón del Valle, Álvarez (1999) reportó un intervalo entre partos de 360 a 390 días; mientras que la tasa de preñez para la ganadería bovina mexicana, basada en razas convencionales, presenta un rango entre 64.3 y $73.9 \%$ (SICEC 2011); valores que son inferiores a los observados en este estudio para las razas criollas, por lo que la mayor capacidad reproductiva de las hembras criollas se considera como una respuesta a la adaptación al medio tropical.

La eficiencia técnica del manejo del hato se refleja en los parámetros productivos y reproductivos, aspectos asociados al nivel tecnológico del sistema de producción y la habilidad de gestión de los ganaderos. Al respecto, la encuesta nacional agropecuaria del 2017 realizada por INEGI, indica que entre las principales prácticas de manejo en los hatos bovinos están la monta controlada (24.8\%), suministro de alimento balanceado (33.5\%) y servicio de asistencia técnica (10.4\%). En el presente estudio se observaron mayores porcentajes para las prácticas de monta controlada y servicio de asistencia técnica, pero menor suministro de alimentos balanceados; debido a la vocación pastoril de las razas criollas. Los productores al gestionar los sistemas de pastoreo incurrieron en un menor uso de fertilizantes, ya que éstos practican principios de ganadería regenerativa. En la alimentación del ganado se incluyó el suministro de forrajes arbustivos de alta calidad, alimento balanceado y sales mineralizadas en la mayor parte de los sistemas. Se observó que los productores realizaron un manejo acorde a la naturaleza dócil de los bovinos criollos con reducida tasa de mortalidad y el aseguramiento de la sustentabilidad de los agroecosistemas implicados.

Es importante mencionar que la información relacionada con el desempeño económico de los sistemas ganaderos es limitada, situación atribuida a la falta o ineficiencia en el registro de información económica de seguimiento del proceso de producción, así como por el desinterés o falta de conocimiento para la estimación de los costos; característica de los ganaderos con escasa visión empresarial. Al respecto, Albarrán-Portillo et al. (2015), reportan que los sistemas de producción bovina de doble propósito del estado de México, bajo condiciones subtropicales, presentan un margen neto de rentabilidad anual de US\$ 9 890. Asimismo, en el estado de Chihuahua los sistemas vaca-becerro desarrollados en pastoreo extensivo, y con finalidad productiva orientada a la obtención de animales destetados para el abasto de carne, presentaron un ingreso promedio por vientre al año de US\$359.08 \pm 174.72 (Callejas-Juárez et al. 2014), valores que son inferiores a lo estimado en el presente estudio. El mayor índice de rentabilidad de los sistemas ganaderos basados en las razas criollas en condiciones del trópico mexicano, está dado por el mayor número de becerros destetados al año, resultado de las tasas de fertilidad y de natalidad sobresalientes propias de estas razas, así como por el menor costo de producción.

\section{CONCLUSIONES}

Los sistemas de producción bovina basados en las razas criollas introducidas Romosinuano y Lechero Tropical, se caracterizaron por la visión empresarial de sus productores, condición diferencial frente a los sistemas tradicionales con razas criollas locales. Los productores presentaron edad avanzada y nivel de educación superior, factores que influyen en el tipo de gestión que ejercen en sus hatos. Los sistemas de producción fueron de tipo pastoril, con presencia destacada de hembras jóvenes y limitada asistencia técnica, características atribuida a la disponibilidad de recursos, estructura del hato y capacidad de gestión del productor. Las razas criollas son una alternativa para el cruzamiento con otras razas predominantes, ya que las primeras tienen la posibilidad de transferir características de interés comercial, así como adaptabilidad a los riesgos del entorno. Es importante mencionar que es necesario aumentar los esfuerzos para priorizar la conservación, preservación y multiplicación de los núcleos 
de bovinos criollos con acciones en las zonas tropicales donde la ganadería exhibe una tendencia hacia la intensificación de los sistemas de producción.

\section{AGRADECIMIENTOS}

Al Tecnológico Nacional de México por en financiamiento al proyecto "Características técnicoeconómicas de los sistemas de producción bovina con biotipos criollos: Directriz para el análisis de sostenibilidad" clave 6462.18. Así como al comité directivo y productores participantes pertenecientes a la Asociación Mexicana de Criadores de Ganado Romosinuano y Lechero Tropical (AMCROLET).

\section{LITERATURA CITADA}

Albarrán-Portillo B, Rebollar-Rebollar S, García-Martínez A, Rojo-Rubio R, Avilés-Nova F, Arriaga-Jordán C (2015) Socioeconomic and productive characterization of dual-purpose farms oriented to milk production in a subtropical region of Mexico. Tropical Animal Health and Production 47: 519-523.

Alonso MR, Ulloa RA (1997) Hacia un proyecto nacional de investigación en genoma de animales domésticos. Veterinaria México 28: 365-370

Álvarez LA (1999) Potencial genético y productivo del ganado Hartón del Valle. Fondo Nacional del Ganado. Instituto Colombiano Agropecuario. Bogotá, Colombia. pp: 94-103. http://hdl.handle.net/20.500.12324/16141. Fecha de consulta 07 de febrero de 2019.

Álvarez LA (2001) Raza Hartón del Valle (HdV). Revista Corpoica 2: 43-48.

Bautista-Martínez Y, Herrera-Haro J, Espinosa-García J, Martínez-Castañeda F, Vaquera-Huerta H, Morales A, et al. (2019) Caracterización económico-productiva del sistema bovino doble propósito en tres regiones tropicales de México. Información Técnica Económica Agraria 115: 134-148.

Burgmaier K, Bullerdieckz P, Gall C (1999) Comportamiento productivo y reproductivo del ganado criollo Reyna en fincas de doble propósito y en lecherías especializadas. Ceiba 40: 279-282.

Callejas-Juárez N, Aranda-Gutiérrez H, Rebollar-Rebollar S, De la Fuente-Martínez M (2014) Situación económica de la producción de bovinos de carne en el estado de Chihuahua, México. Agronomía Mesoamericana 25: 133-139.

Cuevas V, Loaiza A, Espinosa J, Vélez A, Montoya M (2016) Tipología de las explotaciones ganaderas de bovinos doble propósito en Sinaloa, México. Revista Mexicana de Ciencias Pecuarias 7: 69-83.

Cuevas-Reyes V, Rosales-Nieto C (2017) Caracterización del sistema bovino doble propósito en el noroeste de México: productores, recursos y problemática. Revista MVZ Córdoba 23: 6448-6460.

De Alba J (2011) El libro de los bovinos criollos de América. Biblioteca Básica de Agricultura. Colegio de Postgraduados. Montecillo, México: 444p

FAO (2015) The Second Report on the State of the World's Animal Genetic Resources for Food and Agriculture. In Scherf BD and Pilling D (ed). FAO Commission on Genetic Resources for Food and Agriculture Assessments. Rome, Italy. 606p

FAO (2019) Datos sobre producción primaria. http://www.fao.org/faostat/es/data. Fecha de consulta 25 de enero de 2019.

Hernández MP, Estrada FJG, Avilés NF, Yong AG, López GF, Solís MAD, et al. (2013) Tipificación de sistemas campesinos del Sur del Estado de México. México. Universidad y Ciencia 29: 19-31. 
Hernández A, Cervantes P, Goméz-Boucrin, Domínguez B, Barrientos M (2015) Los bovinos criollos en el Golfo de México. En Perezgrovas R, De la Torre F (ed). Los bovinos criollos de México. Chiapas, México. pp: 209-235

INEGI (2017) Encuesta Nacional Agropecuaria 2017. http://www.beta.inegi.org.mx/proyectos/encagro/ena/2017/. Fecha de consulta 25 de enero de 2019.

INEGI (2019) Mapas de climatología de México. https://www.inegi.org.mx/temas/mapas/climatologia/. Fecha de consulta 4 de enero de 2019.

Leos-Rodríguez JA, Serrano-Páez A, Salas-González JM, Ramírez-Moreno PP, Sagarnaga-Villegas M (2008) Caracterización de ganaderos y unidades de producción pecuaria beneficiarios del programa de estímulos a la productividad ganadera (PROGAN) en México. Agricultura, Sociedad y Desarrollo, 5: 213-230

López-Vigoa O, Sánchez-Santana T, Iglesias-Gómez JM, Lamela-López L, Soca-Pérez M, Arece-García J, et al. (2017) Los sistemas silvopastoriles como alternativa para la producción animal sostenible en el contexto actual de la ganadería tropical. Pastos y Forrajes 40: 83-95.

Magaña JG, Ríos G, Martínez JC (2006) Los sistemas de doble propósito y los desafíos en los climas tropicales de México. Archivos Latinoamericanos de Producción Animal 14: 105-114.

Martínez G (1998) El ganado criollo Romosinuano (Romo). AGRI 24: 1-11.

Martínez R, Ramírez R, Núñez R, García J (2018) Parámetros y tendencias genéticas de variables de crecimiento para bovinos Romosinuano en México. Nova Scientia 10: 310-325.

Méndez M, Serrano J, Ávila R, Rosa M, Méndez N (2002) Caracterización morfométrica del bovino criollo mixteco. Archivos de Zootecnia 51: 217-221.

Orantes-Zebadúa MA, Platas-Rosado D, Córdova-Avalos V, De los Santos-Lara MC, Córdova-Avalos A (2014) Caracterización de la ganadería de doble propósito en una región de Chiapas, México. Ecosistemas y Recursos Agropecuarios 1: 49-58

Oros V, Díaz P, Vilaboa J, Martínez J, Torres G (2011) Caracterización por grupos tecnológicos de los hatos ganaderos doble propósito en el municipio de Las Choapas, Veracruz, México. Revista Científica 21: 57-63

Osorio-Arce M, Segura-Correa J, Osorio-Arce D, Marfil-Acevedo A (1999) Caracterización de la ganadería lechera del estado de Yucatán, México. Revista Biomédica 10: 217-227.

Ossa G, Suarez M, Pérez J (2007a) Factores ambientales y genéticos que influyen la edad al primer parto y el intervalo entre partos en hembras de la raza criolla Romosinuano. Revista Corpoica Ciencia y Tecnología Agropecuaria 8: 74-80

Ossa G, Suárez M, Pérez J (2007b) Efectos del medio y la herencia sobre los pesos al nacimiento, al destete y a los 16 meses de edad en terneros de la raza criolla Romosinuano. Ciencia y Tecnología Agropecuaria 8: 81-92.

Parra-Cortés RI, Magaña-Magaña MA, Piñeiro-Vázquez AT (2019) Intensificación sostenible de la ganadería bovina tropical basada en recursos locales: alternativa de mitigación ambiental para América Latina. Revisión Bibliográfica. Información Técnica Económica Agraria. Doi: 10.12706/itea.2019.003

Primo AT (1992) El ganado ibérico en las Américas: 500 años después. Archivos de Zootecnia 41: 421-432.

Rodríguez S, Flores D, León A, Pérez L, Aguilar J (2018) Diagnóstico de sistemas de producción de bovinos para carne en Tejupilco, Estado de México. Revista Mexicana de Ciencias Agrícolas 9: 465-471. 
Rojo-Rubio R, Vázquez-Armijo J, Pérez-Hernández P, Mendoza-Martínez G, Salem A, Albarrán-Portillo B, et al. (2008) Dual purpose cattle production in Mexico. Tropical Animal Health and Production 41: 715-721.

Scheaffer R, Mendenhall W, Ott L (1987) Elementos de muestreo. Grupo Editorial Iberoamérica. México. 58p

Severino-Lendechy VH, Perezgrovas-Garza RA, Montiel-Palacios F, Vilaboa-Arroniz J, Muñoz-González JC, Piñeiro-Vázquez AT (2019) Caracterización del nivel tecnológico y edad al primer estro de hembras Criollo Lechero Tropical. Ecosistemas y Recursos Agropecuarios 6: 353-359.

SICEC (2011) Sistema de Información de Costos, Eficiencia y Competitividad de los Sistemas Pecuarios en México. Informe Bovinos de carne, resultados preliminares de la encuesta 2011. http://www.sicec.unam.mx/ app/webroot/files/archivos_portal/archSISEC209945.pdf. Fecha de consulta 31 de enero de 2019.

Thornton P (2010) Livestock production: recent trends, future prospects. Philosophical Transactions of the Royal Society B: Biological Sciences 365: 2853-2867.

Vance A (2013) Aging farmers not cause for alarm. Feedstuffs. https://www.feedstuffs.com/story-aging-farmerscause-alarm-52-105506. Fecha de consulta 11 de junio de 2019.

Vilaboa J, Díaz P (2009) Caracterización socioeconómica y tecnológica de los sistemas ganaderos en siete municipios del estado de Veracruz, México. Zootecnia Tropical 27: 427-436.

Villaseñor F, De la Torre F, Martínez G, Alvarez H, Perez S, Palacios J, et al. (2017) Caracterización de la respuesta ovarica a la superovulacion en Bovino Criollo Coreño utilizando sosis reducida de FSH. Revista Mexicana de Ciencias pecuarias 8: 225-232

Wright IA, Tarawali S, Blümmel M, Gerard B, Teufel N, Herrero M (2012) Integrating crops and livestock in subtropical agricultural systems. Journal of the Science of Food and Agriculture 92: 1010-1015.

Zavala MG (2019) Los recursos zoogenéticos, ¿Qué son y cuál es su importancia? Universidad Michoacana de San Nicolás de Hidalgo. Michoacán, México. https://www.sabermas.umich.mx/archivo/articulos/14-numero2/30-los-recursos-zoogeneticos-ique-son-y-cual-es-su-importancia.html. Fecha de consulta 4 de enero de 2019. 
